\title{
Selecting a Control Group in Studies of the Familial Coaggregation of Two Disorders: A Quantitative Genetics Perspective
}

\author{
Marc-André Roy, ${ }^{1 *}$ Michael C. Neale, ${ }^{2}$ and Kenneth S. Kendler,3 \\ ${ }^{1}$ Département de Psychiatrie, Centre Hospitalier Robert-Giffard and Centre de Recherche, Université Laval \\ Robert-Giffard, Beauport, Quebec, Canada \\ ${ }^{2}$ Department of Psychiatry, Medical College of Virginia, Richmond, Virginia \\ ${ }^{3}$ Department of Human Genetics, Medical College of Virginia, Richmond, Virginia
}

We sought to compare four different definitions of control groups in studies of the coaggregation between two disorders (A and B) on: 1) their ability to detect valid familial coaggregation; 2) their liability to artifactual evidence for familial coaggregation; and 3) their robustness to the overselection of comorbid cases. Using a quantitative genetic model of transmission, we simulated sibling pairs with familial and nonfamilial sources of comorbidity. Four different definitions of controls were tested to predict disorder $B$ in siblings of cases vs. controls: 1 ) unscreened controls included subjects with $A$ or $B$ as well as subjects with either A or B; 2) in the symmetrical selection method, controls included only subjects without $A$; 3 ) supernormal controls included only subjects without $A$ or $B$; and 4 ) in the pure proband method, cases included subjects with A only, and controls included only subjects without A or B. In the absence of selection bias, 1) the unscreened control and the symmetrical selection methods did not yield spurious evidence for familial coaggregation and could detect familial coaggregation; 2) the supernormal controls yielded spurious evidence of familial coaggregation; and 3 ) the pure proband method sometimes yielded spurious evidence for negative familial coaggregation, and had limited power to detect familial coaggregation. However, the pure proband method was the only one unaffected by overselection of comorbid cases. In the absence of selection bias, both the unscreened control and the symmetrical selection methods are appropriate, and the ro-

\footnotetext{
*Correspondence to: Marc-André Roy, M.D., F.R.C.P., Centre de Recherche Université Laval Robert-Giffard, 2601 de la Canardière, Beauport, Quebec G1J 2G3, Canada. Email: marc-andre. roy@psa.ulaval.ca

Received 26 December 1995; Revised 12 December 1996
}

bustness of the pure proband method to overselection of comorbid cases may be an interesting feature in studies using clinical samples. Moreover, quantitative genetics methods may offer important advantages in the study of familial coaggregation. Am. J. Med. Genet. 74:296-303, 1997.

๑) 1997 Wiley-Liss, Inc.

KEY WORDS: comorbidity; family studies; coaggregation; schizophrenia; depression; anxiety disorders

\section{INTRODUCTION}

Family studies are often used to examine the familial aggregation of disorders. In such studies, cases with A are compared to an "appropriate" comparison group on the risk for A in relatives. Disorder A is said to show familial aggregation if relatives of probands with disorder A have an increased risk for disorder A. Another important use of family studies is to examine the familial coaggregation of two disorders. In such studies, cases with A are compared to an "appropriate" comparison group on the risk for B in relatives. Disorders A and $B$ are said to coaggregate if relatives of probands with disorder A have an increased risk for disorder B. Showing such familial coaggregation may suggest that the same etiologic factors are shared by both disorders. Such investigations of familial coaggregation have been performed for psychiatric disorders such as depression, anxiety disorders, and alcoholism [Weissman, 1990; Merikangas et al., 1994; Roy et al., 1995b], schizophrenia and schizophrenia-spectrum personality disorders [Baron et al., 1985; Kendler et al., 1993c], and various types of cancers [Schildkraut et al., 1989; Parazzini et al., 1992, 1993]. Therefore, finding familial coaggregation (i.e., disorders A and B occurring in different subjects in the same family) may suggest that comorbidity (i.e., disorders $\mathrm{A}$ and $\mathrm{B}$ occurring in the same individual) results from both disorders sharing familial etiologic factors. 
Unfortunately, there is no consensus on what constitutes an appropriate comparison group to study familial coaggregation, since at least four definitions are commonly used. To discuss these approaches, subjects selected because they suffer from A will be referred to as cases, while the comparison group will be referred to as controls. It should be clear that in some situations (e.g., unscreened population controls), the control group may include some subjects with A; however, subjects with A are not selected in the control group specifically because they suffer from that disorder.

First, in the "general population unscreened controls" or ("unscreened controls"), cases include subjects with A, with or without $\mathrm{B}$, and controls include a representative sample of the general population, and therefore do not exclude subjects with A and/or B [Falconer, 1965; Kendler et al., 1993b; Maier et al., 1993]. This method allows the estimation of population prevalence or risk, which is necessary for genetic modeling [Falconer, 1965], since it provides crucial data to estimate the threshold for affection. However, it has been suggested that this method may yield spurious evidence for familial coaggregation if probands with disorder A have a higher risk for disorder B than the general population [Klein, 1993; Weissman et al., 1993]. Consequently, an increased risk for $\mathrm{B}$ in relatives of probands with A would be a spurious consequence of the higher rate of $\mathrm{B}$ in subjects with $\mathrm{A}$.

Second, in the "symmetrical selection" method, cases include subjects with A, with or without $\mathrm{B}$, and controls exclude subjects with disorder A; conversely, subjects with disorder B are not excluded. This is a classical definition for case-control studies, and it has been subjected to the same criticisms as the unscreened controls method. Conversely, some investigators have suggested that the symmetrical selection method yields better power than unscreened controls to uncover familial coaggregation [Wickramaratne, 1995].

Third, in the "supernormal" controls method, cases include subjects with $\mathrm{A}$, with or without $\mathrm{B}$, and controls exclude subjects with any type of psychopathology [Tsuang et al., 1980; Weissman et al., 1993]; therefore, subjects with diagnosis A or B as well as subjects with other disorders are excluded from the control group. This method has been criticized as an example of the "well control" artifact [Schwartz and Link, 1989; Kendler, 1990]. Indeed, a similar screening is not applied to cases and controls, since subjects with B were excluded from the case group but not the control group. As a consequence, rates of "screened-out" disorder (i.e., B) may be increased in relatives of cases vs. "supernormal" controls, producing spurious evidence for coaggregation. Through algebraic development, it was shown that this problem would be more severe with increased prevalence and increased familiality of the disorder screened out [Kendler, 1990].

Fourth, in the "pure proband" strategy, the main contrast compares the risk for $\mathrm{B}$ in relatives of probands with A only to the risk for B in relatives of controls with neither A nor B; a higher risk in relatives of probands with A only would mean that A is genetically related to B [Klein, 1993; Klein and Riso, 1993]. In a second contrast, the risk for $\mathrm{B}$ in relatives of probands with $\mathrm{A}$ and $\mathrm{B}$ is compared to the risk for $\mathrm{B}$ in relatives of probands with A only. From a quantitative genetics perspective, this approach can be criticized for its failing to refer to an etiologic model. Indeed, under a quantitative genetics model of comorbidity, if disorders A and $\mathrm{B}$ share familial factors, their liability is correlated; as a consequence, cases with both $\mathrm{A}$ and $\mathrm{B}$ will have higher liability for both $\mathrm{A}$ and $\mathrm{B}$ than cases with either A or B.

Therefore, there are contradictory statements about the best way to define groups of cases and controls to study familial coaggregation. Three notable features have characterized the debate over this issue so far: 1) the emphasis has been on the risk of observing spurious familial coaggregation; therefore, the power of each method to detect familial coaggregation when present has not received much attention; 2) most contributions have discussed the merits of one or two approaches; we are unaware of any simultaneous discussion and comparison of the four strategies outlined above; and 3) this discussion has been notably atheoretical; indeed, most treatments of the problem have assumed that the disorders analyzed are under dichotomous liability, e.g., they are present or absent, and that they behave as dichotomous variables, while the quantitative genetics perspective suggests that these disorders probably reflect some unmeasured liability distribution [Falconer, 1965; Reich et al., 1975]. For example, in the quantitative genetics perspective, if two disorders share genetic liability, comorbid cases have a higher level of liability than cases with only one of the disorders. Moreover, a quantitative genetics perspective allows for useful distinctions among the sources of comorbidity [Neale and Cardon, 1992]. Indeed, comorbidity may result from the liability to these disorders being influenced by the same genetic or environmental factors, in addition to resulting from methodological artifacts [Caron and Rutter, 1991], including selection biases [Sackett, 1979]. It is then plausible that the optimal definition of cases and controls may vary according to the source of the comorbidity.

This paper will compare how these four strategies to analyze the coaggregation of A and B fare under simulated conditions, based on the following criteria: 1) their ability to detect valid familial coaggregation; 2) their liability to artifactual evidence for familial coaggregation; and 3) their robustness to overselection of comorbid cases.

\section{SIMULATED POPULATIONS}

Quantitative genetics posit that the liability to develop a disorder is influenced by: 1) additive genetic influences resulting from independent effects of multiple genes (A); 2) shared environmental influences resulting from environmental factors to which both members of a pair of relatives have been exposed (C); and 3) nonshared environment (or nonfamilial influences), which are environmental influences specific to each individual (E). Both $\mathrm{A}$ and $\mathrm{C}$ cause pairs of relatives to be more similar than pairs of unrelated individuals, while E causes relatives to be no more correlated than unre- 
lated subjects. Disentangling A and C as explanations for familial resemblance requires adoption or twin data. For the sake of simplicity, we will use a "tau" model, as proposed by Rice et al. [1978], where both A and $\mathrm{C}$, or the transmissible factors, will be referred to as " $F$," for familial factors.

In this tau model, influences from $\mathrm{F}$ and $\mathrm{E}$ add up so that the liability for illness in the population is normally distributed. Individuals whose liability is beyond the threshold $\mathrm{T}_{\mathrm{A}}$ or $\mathrm{T}_{\mathrm{B}}$ develop disorders $\mathrm{A}$ or $\mathrm{B}$, respectively, and individuals below that threshold are unaffected. These thresholds are determined according to the prevalence $\mathrm{P}_{\mathrm{A}}$ and $\mathrm{P}_{\mathrm{B}}$ of the disorders. For example, a $5 \%$ prevalence corresponds to a threshold of 1.64 , which corresponds to the upper $5 \%$ of a normal distribution. The correlations between relatives, i.e., the familial aggregation of disorders $\mathrm{A}$ and $\mathrm{B}$, are $r_{\mathrm{A}}$ and $r_{\mathrm{B}}$, respectively; a correlation of unity means that all the liability is transmitted, while a correlation of 0 means no familial transmission. The degree to which etiologic factors are shared by $\mathrm{A}$ and $\mathrm{B}$ is termed $r_{\mathrm{F}}$, which reflects familial coaggregation, and $r_{\mathrm{E}}$ for the nonfamilial correlation. In most instances [Carey, 1988], a correlation of unity means that the influences are fully shared by both disorders, while a zero correlation means that both disorders have completely distinct determinants.

The simulated populations are composed of sibships including one proband and one sibling. The following parameter values were chosen, based on genetic epidemiological studies suggesting that these values are realistic for psychiatric disorders and traits [Kendler et al., 1993a; Merikangas, et al., 1994; Roy et al., 1995a,b]: 1) for $r_{\mathrm{A}}$ and $r_{\mathrm{B}}$, values were $0.00,0.63$, and 0.80 , corresponding to familiarity $\left(r^{2}\right)$ of $0.00,0.40$, and 0.64 , respectively; 2) for $r_{\mathrm{F}}$, values were $0.00,0.40$, and $0.80 ; 3)$ for $r_{\mathrm{E}}$, values were 0.00 and 0.40 ; and 4) prevalences were set at 0.02 and 0.10 . We ran a total of 104 simulations corresponding to all the relevant permutations. (The total number does not correspond to a straight multiplication of all the possible values for each of the parameter, since some permutations were biologically implausible, e.g., $r_{\mathrm{F}}$ had to be set to 0 whenever either $r_{\mathrm{A}}$ or $r_{\mathrm{B}}$ were equal to 0 .)

The goal of these simulations was to obtain a $4 \times 4$ table describing proband and sibling status for disorders $\mathrm{A}$ and $\mathrm{B}$, for a total of 16 possible combinations. To obtain such a table, the above-mentioned 104 sets of parameters were entered into a transmission model, using the program $\mathrm{Mx}$ [Neale, 1991]. For each of these 104 combinations, the predicted proportion in each of the 16 cells was computed by numerical integration of the multivariate normal distribution, using the program MULNOR [Schervish, 1984]. These integrals, and therefore the predicted cell frequencies, varied according to the $4 \times 4$ correlation matrix of proband's and sibling's liabilities to A and B and according to the thresholds.

The following parameters were not included in the model: 1) sex differences, including scalar, nonscalar, or threshold differences; 2) fertility effect; 3 ) causal relationships, i.e., one disorder causing the other; 4) the type of heterogeneity where comorbid groups identify etiologically distinct disorders (e.g., depression and panic disorder [Weissman et al., 1993]; and 5) diagnostic hierarchy, i.e., in the present case, a diagnosis of A in an individual did not preclude a diagnosis of $B$ in the same individual, and vice versa [Kendler, 1988; Roy and Kendler, 1994].

\section{ANALYSES}

We used the LOGISTIC procedure as implemented in SAS [SAS Institute, 1990], to analyze the relationship between disorders $\mathrm{A}$ and $\mathrm{B}$. The rates of $\mathrm{B}$ in siblings were compared across the four strategies outlined in the introduction: 1) probands with A vs. all probands, notwithstanding their status for or B (unscreened controls); 2) probands with A vs. probands without A (symmetrical selection); 3) probands with A vs. probands with neither A nor B (supernormal); and 4) probands with A only vs. probands with neither A nor B (pure proband).

These simulations were first run without overselection of comorbid cases, i.e., cases were all equally likely to be sampled in the "case group." We then repeated these analyses with the same population of proband and sibling pairs, but introducing a selection bias where comorbid cases (cases with both A and B) were twice as likely to be sampled in the "case group" as cases with either A or B.

The following four qualities for the analyses would be desirable: 1) in the absence of familial coaggregation between $\mathrm{A}$ and $\mathrm{B}$ (i.e., $r_{\mathrm{F}}=0$ ), the analyses would yield no evidence of coaggregation; 2) in the presence of familial coaggregation between $\mathrm{A}$ and $\mathrm{B}$ (i.e., $r_{\mathrm{F}}>0$ ), the analyses would yield evidence for coaggregation; 3 ) the estimate of familial coaggregation would not be influenced by the degree of nonfamilial correlation $\left(r_{\mathrm{E}}\right)$; and 4) the degree of familial coaggregation observed would not be influenced by overselection of comorbid subjects in the case group.

\section{RESULTS}

\section{Step 1}

Tables I and II show the results obtained with the four analytic strategies in the absence of familial coaggregation (i.e., $r_{\mathrm{F}}=0$ ), and assuming no overselection of comorbid subjects in the case group. Therefore, these analyses should yield an odds ratio (OR) of 1 . The unscreened controls and the symmetrical selection methods never yielded any evidence for familial coaggregation and were unaffected by variations in $r_{\mathrm{F}}$. On the other hand, the supernormal control method yielded spurious evidence for familial coaggregation whenever disorder B was transmitted (i.e., $r_{\mathrm{B}}>0$ ), since the ORs in these circumstances were systematically $>1$ (range, 1.03-1.18). A closer inspection of these tables reveals that: 1) the ORs for the supernormal controls were systematically larger in Table II compared to corresponding cells in Table I, indicating that artifactual evidence for coaggregation was stronger with increasing degree of familiality of A and B; 2) the ORS for the supernormal controls were systematically larger with increasing prevalence of disorder $\mathrm{A}$; and 3) the ORs for the 
TABLE I. Analyses of Coaggregation of A and B in the Absence of Familial Coaggregation $\left(r_{\mathrm{F}}=0.00\right)$, and With Lower Values of Familial Aggregation for Both Disorders $\left(r_{\mathrm{A}}\right.$ and $\left.r_{\mathrm{B}}=0.63\right)$

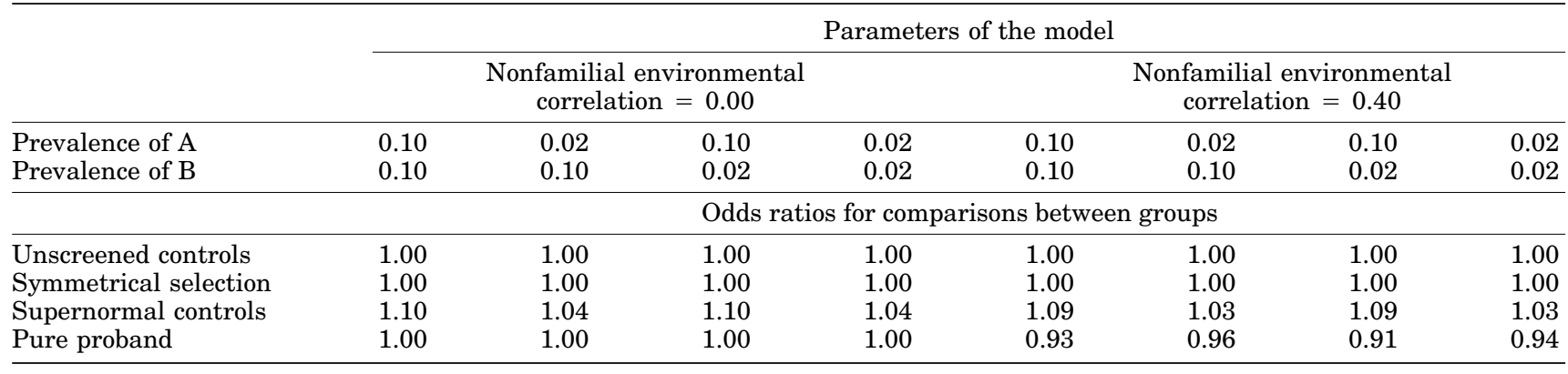

supernormal controls were larger with a null nonfamilial correlation $\left(r_{\mathrm{E}}=0\right)$ between $\mathrm{A}$ and B than with $r_{\mathrm{E}}$ $=0.40$. Finally, the pure proband method did not yield spurious evidence of positive familial coaggregation, but it yielded spurious evidence for negative familial coaggregation (i.e., lower risk of $\mathrm{B}$ in relatives with $\mathrm{A}$ than in controls with neither A nor B) whenever $r_{\mathrm{E}}>0$.

\section{Step 2}

Tables III-VI report the results using the four analytic paradigms in the presence of familial coaggregation (i.e., $r_{\mathrm{F}}>0$ ) and assuming no overselection of comorbid subjects in the case group. Therefore, the analyses should yield ORs $>1$. These tables reveal that all four methods met that criterion and yielded ORs $>1$. However, the magnitude of the ORs varied across the methods, with the following order of increasing ORs in 26 out of 32 permutations: 1 ) pure proband method; 2 ) unscreened controls; 3) symmetrical selection; and 4) supernormal strategy. In six permutations, the order between 1) and 2) was reversed; these corresponded to situations where the prevalence of $B$ was larger than that of A. Among these four methods, only the supernormal method was affected by variations in $r_{\mathrm{E}}$, but the changes in the ORs were minimal, never exceeding 0.05 .

\section{Step 3}

Table VII shows the results when we repeated the simulation reported in Table II, but introducing a selection bias where comorbid cases were twice as likely than noncomorbid cases to be sampled in the case group. Therefore, comparing ORs in Table VII to corresponding ORs in Table II reflects how much these four models are influenced by such a selection bias; again, the extent to which the ORs are $>1$ reflects spurious evidence for familial coaggregation. Table VII reveals that both the unscreened controls and the symmetrical selection methods yielded similar ORs that were systematically $>1$, while these methods never yielded spurious evidence of familial coaggregation in the absence of this selection bias (Table II). Moreover, the bias introduced by the supernormal control method was made worse than before, with ORs in Table VII going as high as 1.38. For all of these three methods, the overestimation of ORs was made worse with $r_{\mathrm{E}}=$ 0.40. Finally, the pure proband method was unaffected by the introduction of selection bias, as it yielded ORs similar to those reported in Table II.

\section{Step 4}

Table VIII shows the results when we repeated the simulation reported in Table VI, but introducing a selection bias where comorbid cases were twice as likely as noncomorbid cases to be sampled in the case group. Therefore, comparing Table VIII to Table VI reflects the impact of this selection bias. The selection bias resulted in increased ORs for the unscreened control, the symmetrical selection, and the supernormal strategies. On the other hand, the pure proband method yielded ORs exactly similar to those previously obtained.

\section{DISCUSSION}

\section{Performance of the Methods in the Absence of Overselection of Comorbid Cases}

In the absence of overselection of comorbid cases, the symmetrical selection and the unscreened control

TABLE II. Analyses of Coaggregation of A and B in the Absence of Familial Coaggregation $\left(r_{\mathrm{F}}=0.00\right)$, and With Higher Values of Familial Aggregation for Both Disorders $\left(r_{\mathrm{A}}\right.$ and $\left.r_{\mathrm{B}}=0.80\right)$

\begin{tabular}{|c|c|c|c|c|c|c|c|c|}
\hline \multirow[b]{3}{*}{ Prevalence of $\mathrm{A}$} & \multicolumn{8}{|c|}{ Parameters of the model } \\
\hline & \multicolumn{4}{|c|}{$\begin{array}{c}\text { Environmental } \\
\text { correlation }=0.00\end{array}$} & \multicolumn{4}{|c|}{$\begin{array}{c}\text { Environmental } \\
\text { correlation }=0.40\end{array}$} \\
\hline & 0.10 & 0.02 & 0.10 & 0.02 & 0.10 & 0.02 & 0.10 & 0.02 \\
\hline Prevalence of B & \multicolumn{8}{|c|}{ Comparisons between groups } \\
\hline Unscreened controls & 1.00 & 1.00 & 1.00 & 1.00 & 1.00 & 1.00 & 1.00 & 1.00 \\
\hline Pure proband & 1.00 & 1.00 & 1.00 & 1.00 & 0.93 & 0.96 & 0.91 & 0.94 \\
\hline
\end{tabular}


TABLE III. Analyses of Coaggregation of A and B in the Presence of Moderate Familial Coaggregation $\left(r_{\mathrm{F}}=0.40\right)$, and With Lower Values of Familial Aggregation for Both Disorders $\left(r_{\mathrm{A}}\right.$ and $\left.r_{\mathrm{B}}=0.63\right)$

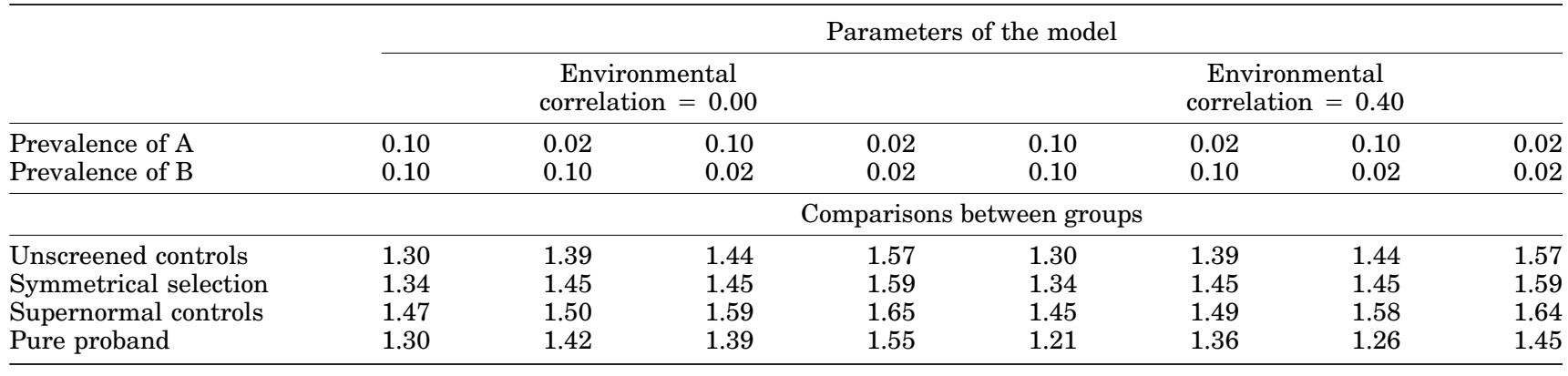

methods did not yield artifactual evidence for familial aggregation. However, the symmetrical selection method offered better power than the unscreened control method to detect familial aggregation when present. This better power resulted from the exclusion of cases with A from the control group in the symmetrical control method; when the familial liability for A and B was correlated, excluding cases with A resulted in a lower mean familial liability for B in controls. Both methods were criticized because it was posited that if probands with $\mathrm{A}$ are at higher risk for $\mathrm{B}$ that the population prevalence, then the higher risk for $\mathrm{B}$ in their relatives would be spurious and would not reflect real familial coaggregation [Klein, 1993]. However, our simulations suggest that when the higher rate for $\mathrm{B}$ in probands with A does not result from overselection of comorbid cases, but instead results from both disorders sharing etiologic influences, an elevation of the risks for $B$ in relatives of probands with A actually reflects the familial correlation between A and B.

While the unscreened population control approach offered slightly less power than the symmetrical selection method to detect valid familial coaggregation, unscreened controls selected from the general population offer the following advantages: 1 ) they may yield an estimate of the rate of comorbidity in the general population; 2) they estimate the prevalence of a disorder in the population, and this parameter is necessary to estimate thresholds in genetic modeling, and eases comparisons with other samples; and 3) they allow for flexibility in running different contrasts. For example, in a study including different groups of probands (e.g., schizophrenic, schizoaffective, bipolar, and schizophrenia spectrum personality disorders (SSPD)), the inves- tigators may be interested in the coaggregation of various disorders. In such an example, the interest may be a comparison of the rates of depression in relatives of SSPD probands vs. those of probands with depression; in this case, using the symmetrical selection method, SSPD controls could be excluded. Alternatively, the rates of depression in relatives of bipolar subjects vs. those of control subjects could also be compared; in this situation, using the symmetrical selection method, controls with bipolar disorder could be excluded.

The supernormal control approach was shown to yield spurious evidence of familial coaggregation, as previously described by Kendler [1990]. Indeed, the results obtained through the present simulation fitted perfectly with those reported in figures of that article. However, the present study yielded more modest biases than those reported in Kendler [1990]. To better understand this apparent discrepancy, we compared the parameters used in both articles. This comparison revealed that the present work used a narrower range of parameters, because the quantitative genetics model used in the present study allowed setting biologically realistic boundaries to the parameters used. Using ORs, such as was done in the previous article, does not lead to such straightforward boundaries (see General Comment, below, for a more detailed discussion of the effect of using ORs).

The pure proband method yielded evidence for negative coaggregation in the absence of any coaggregation, whenever $r_{\mathrm{E}}=0.40$. In this case, because of the correlation in environmental factors, comorbid probands have, on average, a higher environmental liability for both disorders than noncomorbid probands. Moreover, the pure proband method offered limited power to de-

TABLE IV. Analyses of Coaggregation of A and B in the Presence of Strong Familial Coaggregation $\left(r_{\mathrm{F}}=0.40\right)$, and With Lower Values of Familial Aggregation for Both Disorders $\left(r_{\mathrm{A}}\right.$ and $\left.r_{\mathrm{B}}=0.80\right)$

\begin{tabular}{|c|c|c|c|c|c|c|c|c|}
\hline \multirow{2}{*}{ Prevalence of A } & \multicolumn{8}{|c|}{ Parameters of the model } \\
\hline & \multicolumn{4}{|c|}{$\begin{array}{c}\text { Environmental } \\
\text { correlation }=0.00\end{array}$} & \multicolumn{4}{|c|}{$\begin{array}{c}\text { Environmental } \\
\text { correlation }=0.40\end{array}$} \\
\hline \multirow[t]{2}{*}{ Prevalence of B } & 0.10 & 0.10 & 0.02 & 0.02 & 0.10 & 0.10 & 0.02 & 0.02 \\
\hline & \multicolumn{8}{|c|}{ Comparisons between groups } \\
\hline Unscreened controls & 1.51 & 1.66 & 1.76 & 2.01 & 1.51 & 1.66 & 1.76 & 2.01 \\
\hline Pure proband & 1.46 & 1.69 & 1.59 & 1.90 & 1.35 & 1.61 & 1.42 & 1.76 \\
\hline
\end{tabular}


TABLE V. Analyses of Coaggregation of A and B in the Presence of Strong Familial Coaggregation $\left(r_{\mathrm{F}}=0.80\right)$, and With Lower Values of Familial Aggregation for Both Disorders $\left(r_{\mathrm{A}}\right.$ and $\left.r_{\mathrm{B}}=0.63\right)$

\begin{tabular}{|c|c|c|c|c|c|c|c|c|}
\hline \multirow{2}{*}{ Prevalence of $\mathrm{A}$} & \multicolumn{8}{|c|}{ Parameters of the model } \\
\hline & \multicolumn{4}{|c|}{$\begin{array}{c}\text { Environmental } \\
\text { correlation }=0.00\end{array}$} & \multicolumn{4}{|c|}{$\begin{array}{c}\text { Environmental } \\
\text { correlation }=0.40\end{array}$} \\
\hline Prevalence of B & \multicolumn{8}{|c|}{ Comparisons between groups } \\
\hline Unscreened controls & 1.66 & 1.86 & 2.01 & 2.36 & 1.66 & 1.86 & 2.01 & 2.36 \\
\hline Pure proband & 1.69 & 1.98 & 1.91 & 2.31 & 1.78 & 1.92 & 1.76 & 2.18 \\
\hline
\end{tabular}

tect familial coaggregation, yielding smaller ORs than the other analytical methods in the presence of familial coaggregation. Another disadvantage of this method is that it would require screening out a substantial proportion of probands with A who also suffer from B. For example, in a recent twin study of generalized anxiety disorder (GAD) and major depression (MD) [Roy et al., $1995 \mathrm{~b}$, less than half of the subjects in the general population with GAD did not suffer from MD. Such a high rate of comorbidity would make recruiting a group of probands with "pure" GAD quite difficult.

In summary, in the absence of selection bias, the symmetric selection and the unscreened control methods were both appropriate and offered different advantages, while the supernormal control and the pure proband methods both suffered from important flaws.

\section{Performance of the Methods Under Overselection of Comorbid Cases}

The overselection of comorbid cases in the case group had important effects on the unscreened control, the symmetrical selection, and the supernormal control methods. Indeed, with overselection of comorbid cases, these three methods provided spurious evidence for familial coaggregation, with the supernormal control method being the most severely affected. Conversely, only the pure proband method was not affected. This resulted from cases being already selected for their comorbidity under the pure proband method. Therefore, overselecting comorbid cases simply increased the proportion of cases in the comorbid proband group, but did not affect the rate of disorders A or B in relatives.

Such overselection is particularly likely in studies based on clinically ascertained cases; at least two sources can be distinguished. First, in Berkson's bias [Berkson, 1946], it is assumed that the probability of seeking treatment for one disorder is independent from the status for the other one. For example, if subjects with either disorder A or B have a $20 \%$ probability of seeking treatment, and if the probability of seeking treatment for either disorder is independent from the presence of the other, subjects with both $A$ and $B$ would have a $36 \%$ probability of seeking treatment, compared to $20 \%$ for subjects with either A or B, resulting in an overrepresentation of subjects with both A and B in clinical samples. Second, in the "clinical bias," subjects with both disorders A and B are more likely to seek treatment because they would be more incapacitated. In the preceding example, an investigator could observe a $50 \%$ probability of seeking treatment, which is more than the $36 \%$ rate predicted under the independence of the effect; the difference between $50 \%$ and $36 \%$ reflects how much both disorders interact in increasing the risk of seeking treatment [Galbaud du Fort et al., 1993].

Such biases are unlikely to arise if the vast majority of cases with the disorder seek treatment and are recorded through some register [Walsh et al., 1980; Kendler et al., 1993b]. For disorders for which the probability of seeking treatment is much less than $100 \%$ (e.g., major depression, anxiety disorders), the Berkson and clinical biases are much more likely, and the best way to avoid them would be to select a population sample. However, getting large enough samples for disorders that are not highly prevalent (e.g., anorexia nervosa, obsessive compulsive disorder) may prove an excessive burden. For such disorders, investigators may have to rely on clinical samples; strategies to protect against

TABLE VI. Analyses of Coaggregation of A and B in the Presence of Strong Familial Coaggregation $\left(r_{\mathrm{F}}=0.80\right)$, and With Higher Values of Familial Aggregation for Both Disorders $\left(r_{\mathrm{A}}\right.$ and $\left.r_{\mathrm{B}}=0.80\right)$

\begin{tabular}{|c|c|c|c|c|c|c|c|c|}
\hline \multirow[b]{3}{*}{ Prevalence of A } & \multicolumn{8}{|c|}{ Parameters of the model } \\
\hline & \multicolumn{4}{|c|}{$\begin{array}{c}\text { Environmental } \\
\text { correlation }=0.00\end{array}$} & \multicolumn{4}{|c|}{$\begin{array}{c}\text { Environmental } \\
\text { correlation }=0.40\end{array}$} \\
\hline & 0.10 & 0.02 & 0.10 & 0.02 & 0.10 & 0.02 & 0.10 & 0.02 \\
\hline Prevalence of B & \multicolumn{8}{|c|}{ Comparisons between groups } \\
\hline Unscreened controls & 2.19 & 2.55 & 2.96 & 3.66 & 2.19 & 2.55 & 2.96 & 3.66 \\
\hline Pure proband & 2.13 & 2.77 & 2.51 & 3.36 & 1.99 & 2.66 & 2.27 & 3.13 \\
\hline
\end{tabular}


TABLE VII. Analyses of Coaggregation of A and B in the Absence of Familial Coaggregation $\left(r_{\mathrm{F}}=0.00\right)$, With Higher Values of Familial Aggregation for Both Disorders $\left(r_{\mathrm{A}}\right.$ and $\left.r_{\mathrm{B}}=0.80\right)$ and With Selection Bias*

\begin{tabular}{|c|c|c|c|c|c|c|c|c|}
\hline \multirow{4}{*}{$\begin{array}{l}\text { Prevalence of A } \\
\text { Prevalence of B }\end{array}$} & \multicolumn{8}{|c|}{ Parameters of the model } \\
\hline & \multicolumn{4}{|c|}{$\begin{array}{c}\text { Environmental } \\
\text { correlation }=0.00\end{array}$} & \multicolumn{4}{|c|}{$\begin{array}{c}\text { Environmental } \\
\text { correlation }=0.40\end{array}$} \\
\hline & 0.10 & 0.02 & 0.10 & 0.02 & 0.10 & 0.02 & 0.10 & 0.02 \\
\hline & 0.10 & 0.10 & 0.02 & 0.02 & 0.10 & 0.10 & 0.02 & 0.02 \\
\hline & \multicolumn{8}{|c|}{ Comparisons between groups } \\
\hline Unscreened controls & 1.13 & 1.07 & 1.13 & 1.07 & 1.16 & 1.10 & 1.17 & 1.12 \\
\hline Symmetrical selection & 1.13 & 1.07 & 1.13 & 1.07 & 1.16 & 1.10 & 1.17 & 1.12 \\
\hline Supernormal controls & 1.33 & 1.16 & 1.33 & 1.16 & 1.36 & 1.19 & 1.38 & 1.20 \\
\hline Pure proband & 1.00 & 1.00 & 1.00 & 1.00 & 0.93 & 0.96 & 0.91 & 0.94 \\
\hline
\end{tabular}

*Through selection bias, cases with both A and B were twice as likely as cases with either A or B to be selected in the case group.

selection biases are needed. In these situations, the symmetrical selection and the unscreened control methods may be appropriate if the rate of comorbidity in the community is known, allowing to correct for these selection biases. Unfortunately, while estimates of rates of comorbidity in the community are available [Regier et al., 1990], they may not apply to the particular population under study, since such rates may vary across studies depending on factors such as diagnostic criteria, hierarchy rules, and diagnostic instruments, which make the "pure proband" method a potentially interesting solution in such instances.

\section{The Issue of Spectrum Disorders}

A quantitative genetics perspective on the selection of controls may bear important implications for disorders which have a genetic correlation of unity with the disorder under study. For example, there is significant evidence that schizophrenia and SSPD share similar genetic determinants [Kendler et al., 1995]. A consequence of the present line of reasoning would be that to study the coaggregation of depression in relatives of schizophrenic probands, if using the symmetrical selection method, it would be correct to exclude SSPD subjects from the comparison group. However, such exclusions should be used only if a genetic correlation of unity between these disorders is firmly established. Otherwise, such exclusions could result in a supernormal artifact.

\section{General Comment}

The present results may also underlie potential limitations in using ORs to quantify coaggregation of two disorders. Indeed, ORs do not translate easily into biological terms; conversely, the genetic correlation vary from 0 to 1 , which is biologically meaningful. For example, in our simulations, using the symmetrical selection, setting a genetic correlation $\left(r_{\mathrm{F}}\right)$ of 0.8 , ORS varied from 1.78-3.87, depending on the degree of familial aggregation of each disorder and its prevalence. This effect of the prevalence of a disorder on the magnitude of the OR is also found when ORs are used to quantify familial aggregation [Reich et al., 1975; Kendler, 1989]. This range of ORs may give a false impression of very different degrees of sharing familial factors. Given these limitations, we suggest considering the use of path analyses to investigate the coaggregation of two or more disorders [Kendler et al., 1992; Merikangas et al., 1994].

However, we do not contend that ORs should not be used to quantify familial coaggregation. Indeed, they offer advantages, i.e., 1) they are easier than quantitative genetic parameters to translate into actual risks for relatives of affected subjects; 2 ) in some instances, it is easier to get an intuitive understanding of ORs than of quantitative genetic parameters; and 3) using a quantitative genetics model is appropriate for traits showing polygenic inheritance, but not for other modes of transmission. Conversely, analyses using ORs make no assumption about the mode of inheritance.

\section{STUDY LIMITATIONS}

The present report paper should be interpreted while keeping in mind the following limitations.

First, the multifactorial model is only one among

TABLE VIII. Analyses of Coaggregation of A and B in the Presence of Strong Familial Coaggregation $\left(r_{\mathrm{F}}=0.80\right)$, With Higher Values of Familial Aggregation for Both Disorders $\left(r_{\mathrm{A}}\right.$ and $\left.r_{\mathrm{B}}=0.80\right)$ and Selection Bias*

\begin{tabular}{|c|c|c|c|c|c|c|c|c|}
\hline \multirow[b]{3}{*}{ Prevalence of $\mathrm{A}$} & \multicolumn{8}{|c|}{ Parameters of the model } \\
\hline & \multicolumn{4}{|c|}{$\begin{array}{c}\text { Environmental } \\
\text { correlation }=0.00\end{array}$} & \multicolumn{4}{|c|}{$\begin{array}{c}\text { Environmental } \\
\text { correlation }=0.40\end{array}$} \\
\hline & 0.10 & 0.02 & 0.10 & 0.02 & 0.10 & 0.02 & 0.10 & 0.02 \\
\hline Prevalence of B & \multicolumn{8}{|c|}{ Comparisons between groups } \\
\hline Unscreened controls & 2.47 & 2.82 & 3.29 & 4.13 & 2.45 & 2.85 & 3.20 & 4.15 \\
\hline Pure proband & 2.13 & 2.77 & 2.51 & 3.36 & 1.99 & 2.66 & 2.27 & 3.13 \\
\hline
\end{tabular}

*Through selection bias, cases with both A and B were twice as likely as cases with either A or B to be selected in the case group. 
competing models to explain the etiology of psychiatric disorders. Other models of transmission could lead to different choices of control groups.

Second, we deliberately chose a simplistic model, and failed to model the factors listed at the end of the description of the simulation. While this choice has heuristic value, other artifactual sources of comorbidity such as those discussed in Caron and Rutter [1991] should be considered in analyzing data.

\section{ACKNOWLEDGMENTS}

M.-A.R. was supported by a fellowship grant from the McLaughlin Foundation (from September 1992May 1994), and by a Career Development Award from the Fonds de la Recherche en Santé du Québec (from July 1995). The authors thank Dr. Chantal Caron, M.D., M.Sc., F.R.C.P., and Dr. Stéphane Bouchard, Ph.D., for useful comments on this manuscript.

\section{REFERENCES}

Baron M, Gruen R, Asnis L, Lord S (1985): Familial transmission of schizotypal and borderline personality disorders. Am J Psychiatry 142:927934.

Berkson J (1946): Limitations of the application of fourfold table analysis to hospital data. Biometrics Bull 2:47-53.

Carey G (1988): Inference about genetic correlations. Behav Genet 18:329_ 338.

Caron C, Rutter M (1991): Comorbidity in child psychopathology: Concepts, issues and research strategies. J Child Psychol Psychiatry 32: 1063-1080.

Falconer DS (1965): The inheritance of liability to certain diseases, estimated from the incidence among relatives. Ann Hum Genet 29:51-76.

Galbaud du Fort G, Newman SC, Bland RC (1993): Psychiatric comorbidity and treatment seeking. Sources of selection bias in the study of clinical populations. J Nerv Ment Dis 181:467-474.

Kendler KS (1988): The impact of diagnostic hierarchies on prevalence estimates for psychiatric disorders. Compr Psychiatry 29:218-227.

Kendler KS (1989): Limitations of the ratio of concordance rates in monozygotic and dizygotic twins. Arch Gen Psychiatry 46:477-478.

Kendler KS (1990): The super-normal control group in psychiatric genetics: Possible artifactual evidence for coaggregation. Psychiatr Genet 1:4553.

Kendler KS, Neale MC, Kessler RC, Heath AC, Eaves LJ (1992): Major depression and generalized anxiety disorder: Same genes, (partly) different environments? Arch Gen Psychiatry 49:716-722.

Kendler KS, Heath AC, Neale MC, Kessler RC, Eaves LJ (1993a): Alcoholism and major depression: A twin study of the causes of comorbidity. Arch Gen Psychiatry 50:690-698.

Kendler KS, McGuire M, Gruenberg AM, O'Hare A, Spellman M, Walsh D (1993b): The Roscommon Family Study: I. Methods, diagnosis of probands and risk of schizophrenia in relatives. Arch Gen Psychiatry 50: 527-540.

Kendler KS, McGuire M, Gruenberg AM, O'Hare A, Spellman M, Walsh D (1993c): The Roscommon Family Study: III. Schizophrenia-related personality disorders in relatives. Arch Gen Psychiatry 50:781-788.

Kendler KS, Neale MC, Walsh D (1995): Evaluating the spectrum concept of schizophrenia in the Roscommon Family Study. Am J Psychiatry 152:749-754.
Klein DF (1993): The utility of the super-normal control group in psychiatric genetics. Psychiatr Genet 3:17-19.

Klein DN, Riso LP (1993): Psychiatric disorders: Problems of boundaries and comorbidity. In Costello CG (ed): "Basic Issues in Psychopathology." New York: Guilford Press, pp 19-66.

Maier W, Lichtermann D, Minges J, Hallmayer J, Heun R, Benkert O, Levinson DF (1993): Continuity and discontinuity of affective disorders and schizophrenia. Arch Gen Psychiatry 50:871-883.

Merikangas KR, Risch NJ, Weissman MM (1994): Comorbidity and cotransmission of alcoholism, anxiety and depression. Psychol Med 24: 69-80.

Neale M (1991): Statistical modelling with MX. Medical College of Virginia, Richmond, VA.

Neale M, Cardon LR (1992): "Methodology for Genetic Studies of Twins and Families." Dordrecht: Kluwer Academic Publishers, pp 231-259.

Parazzini F, Negri E, La Vecchia C, Restelli C, Franceschi S (1992): Family history of reproductive cancers and ovarian cancer risk: An Italian case-control study. Am J Epidemiol 135:35-40.

Parazzini F, La Vecchia C, Negri E, Franceschi S, Tozzi L (1993): Family history of breast, ovarian and endometrial cancer and risk of breast cancer. Int J Epidemiol 22:614-618.

Regier DA, Burke JD, Burke KC (1990): Comorbidity of affective and anxiety disorders in the NIMH Epidemiologic Catchment Area Program. In Maser JD, Cloninger CR (eds): "Comorbidity of Mood and Anxiety Disorders." Washington, DC: American Psychiatric Press, pp 113-122.

Reich T, Cloninger CR, Guze SB (1975): The multifactorial model of disease transmission: I. Description of the model and its use in psychiatry. $\mathrm{Br}$ J Psychiatry 127:1-10.

Rice J, Cloninger RC, Reich T (1978): Multifactorial inheritance with cultural transmission and assortative mating. I. Description and basic properties of the unitary models. Am J Hum Genet 30:618-643.

Roy M-A, Kendler KS (1994): The effect of diagnostic hierarchy in geneticepidemiological studies of psychiatric disorders. Arch Gen Psychiatry 51:926-927.

Roy M-A, Neale M, Kendler KS (1995a): The genetic epidemiology of selfesteem. Br J Psychiatry 166:813-820.

Roy M-A, Neale MC, Kendler KS (1995b): A twin study of generalized anxiety disorder and major depression. Psychol Med 25:1037-1049.

Sackett DL (1979): Bias in analytic research. J Chronic Dis 32:51-63.

SAS Institute (1990): "SAS/STAT User's Guide, 6th Edition." Cary, NC: SAS Institute, Inc.

Schervish MJ (1984): Multivariate normal probability with error bounded. Appl Stat 33:81-94.

Schildkraut JM, Risch N, Thompson WD (1989): Evaluating genetic association among ovarian, breast and endometrial cancer: Evidence for a breast/ovarian cancer relationship. Am J Hum Genet 45:521-529.

Schwartz S, Link BG (1989): The "well control" artifact in case/control studies of specific psychiatric disorders. Psychol Med 19:737-742.

Tsuang MT, Winokur G, Crowe RR (1980): Morbidity risks of schizophrenia and affective disorders among first degree relatives of patients with schizophrenia, mania, depression and surgical controls. Am J Psychiatry 137:497-504.

Walsh D, O'Hare A, Blake B, Halpenny JV, O'Brien PF (1980): The treated prevalence of mental illness in the Republic of Ireland-The Three County Case Register Study. Psychol Med 10:465-470.

Weissman MM (1990): Evidence for comorbidity for anxiety and depression: Review of family and genetic studies of children. In Maser JD, Cloninger RC (eds): "Comorbidity of Mood and Anxiety Disorders." Washington, DC: American Psychiatric Press, pp 349-365.

Weissman MM, Wickramaratne P, Adams PE, Lish LD, Horwath E, Charney D, Woods SW, Leeman E, Frosch E (1993): The relationship between panic disorder and major depression: A new family study. Arch Gen Psychiatry 50:767-780.

Wickramaratne PJ (1995): Selecting control groups for studies of familial aggregation of disease. J Clin Epidemiol 48:1019-1029. 